\title{
TorchMinusOne: A Tool for Deterministic Geophysical Inversion
}

\author{
SHERVIN MANZURI SHALMANI ${ }^{1}$, PEJMAN \\ SHAMSIPOUR $^{1}$, AMIN AGHAEE ${ }^{1}$ AND GRACE DUPUIS ${ }^{2}$ \\ ${ }^{1}$ Goldspot Discoveries Corp. \\ 2 ...
}

Presenting Author: shervin.manzurishalmani@goldspot.ca

Inversion algorithms are used to recover models of the Earth's 3D physical properties such as density and conductivity such that anomalies in 2D ground-truth geophysical survey data are adequately reproducible. However, due to the survey data's inherent uncertainty, the solution to the inverse problem is nonunique. Deterministic inversion techniques are designed to ignore uncertainty and take the most likely solution at each iteration; nevertheless, in contrast to stochastic inversion, deterministic inversion is much lighter in terms of computational costs and can incorporate uncertainty through posterior procedures. This computational simplicity makes the usage of deterministic inversion lucrative, especially in today's world where massive datasets are efflorescent. Nevertheless, while much faster than stochastic inversion, many complex matrix multiplication operations are performed in deterministic inversion, which is slower on a CPU.

In this work, we implement a fast, GPU based deterministic inversion of geophysical data using mini-batch gradient descent as a part of the MinusOne inversion framework. To the best of our knowledge, this is the first work implementing a GPU based deterministic inversion tool using PyTorch that can handle both model optimization and GPU operations. We take advantage of the fast matrix multiplication capabilities of CUDA inside the PyTorch framework. It should be noted that while significant speedups are achieved through GPU based operations, we should carefully consider GPU memory limits. As such, we currently store mini-batches of the sensitivity matrix outside the GPU. We also believe that this tool must also implement different types of regularization and constraints. We achieve up to $15 \mathrm{X}$ speedups compared to CPU-based deterministic inversion, using synthetic datasets with close to real-life sizes, and we plan to do a case study on real data. 